\title{
Behavioral Mechanisms
}

National Cancer Institute

\section{Source}

National Cancer Institute. Behavioral Mechanisms. NCI Thesaurus. Code C15996.

Studies that develop and test specific interventions aimed at changing negative health behaviors. 